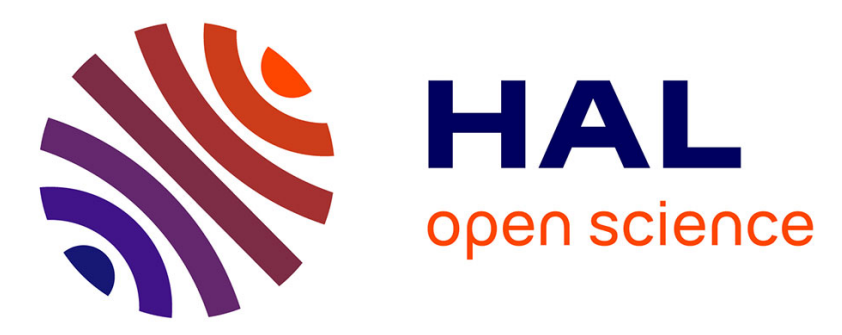

\title{
Estimation of Foetal Contribution to ECG Recordings Using Oblique Projection Technique Exploiting Cyclostationary Properties of the Heartbeat Signals
}

\author{
Julien Roussel, Michel Haritopoulos
}

\section{- To cite this version:}

Julien Roussel, Michel Haritopoulos. Estimation of Foetal Contribution to ECG Recordings Using Oblique Projection Technique Exploiting Cyclostationary Properties of the Heartbeat Signals. MBEC2014 - 6th European Conference of the International Federation for Medical and Biological Engineering, Sep 2014, DubrovniK, Croatia. pp.4 Pages. hal-01003851

\section{HAL Id: hal-01003851 \\ https://hal.science/hal-01003851}

Submitted on 10 Sep 2014

HAL is a multi-disciplinary open access archive for the deposit and dissemination of scientific research documents, whether they are published or not. The documents may come from teaching and research institutions in France or abroad, or from public or private research centers.
L'archive ouverte pluridisciplinaire $\mathbf{H A L}$, est destinée au dépôt et à la diffusion de documents scientifiques de niveau recherche, publiés ou non, émanant des établissements d'enseignement et de recherche français ou étrangers, des laboratoires publics ou privés. 


\title{
Estimation of Foetal Contribution to ECG Recordings Using Oblique Projection Technique Exploiting Cyclostationary Properties of the Heartbeat Signals
}

\author{
J. Roussel ${ }^{1}$ and M. Haritopoulos ${ }^{1}$ \\ ${ }^{1}$ Univ. Orléans, ENSI de Bourges, PRISME Laboratory, EA 4229, F28000, Chartres, France
}

\begin{abstract}
In this paper, the authors propose to estimate the contribution of the electrical activity of the foetal heart to each component of cutaneous electrocardiogram (ECG) recordings using an oblique projection technique. To date, related research work reported in the literature using Blind Source Separation (BSS) or Independent Component Analysis (ICA) methods rely on a widely used projection techniques family, which is the orthogonal projection. More recently, reported work in other areas of biomedical research is based on the use of oblique projection techniques in order to remove the artifacts from the available recordings and to estimate the contribution of the Signal of Interest (SoI) to the data set. The novel approach presented in this paper is tailored to the problem of foetal ECG (FECG) contribution estimation. The optimal steering vector's computation procedure for the oblique projection is based on the use of the cyclostationary properties of the SoI and on the concept of Multidimensional Independent Component Analysis (MICA). The latter is used to gather into independent subspaces the components estimated using a BSS/ICA method and corresponding to the foetus' heartbeats, the mother's heartbeats and various artifacts and noise sources. Early comparative results with the orthogonal approach obtained after application of both methods to synthetic non-invasive FECG recordings illustrate the reliability as well as the effectiveness of the proposed method. A discussion and perspectives for future research conclude this work.
\end{abstract}

Keywords - foetal electrocardiogram, oblique projection, principal component analysis, multidimensional independent component analysis, cyclic coherence

\section{INTRODUCTION}

Foetal heart monitoring using non-invasive techniques is a valuable tool for use by clinicians as it helps establishing an early diagnosis that can prevent complications due to heart defects of the foetus. The electrical activity of the foetal heart is measured by means of cutaneous electrodes applied on the mother's skin. The main difficulties arising in this case are the relatively low amplitude voltage of the foetal electrocardiogram (FECG) compared to the maternal one (MECG) and the contamination of the FECG with other interferences and noise sources, such as maternal respiration, random instrumentation noise, baseline wandering or even foetal movements.

Among the first attempts to extract the FECG from multilead MECG recordings, one may mention [1] were the authors used the Gram-Schmidt technique to build a set of orthogonal basis functions representing the thoracic MECG signals assumed independent and appropriately represented by a dipole; finally, and following a "cancellation" system, the foetal contribution is extracted by subtracting the maternal one from the thoracic MECG recordings. Since then, and especially these last decades, the emergence of new signal processing techniques has paved the way for new research effort devoted to the problem of foetal ECG extraction.

More particularly, Blind Source Separation (BSS) techniques [2] and the higher-order-statistics (HOS)-based class of methods known as Independent Component Analysis (ICA) [3] have been successfully applied to various biomedical problems, such as artifacts removal from electroencephalographic (EEG) or electromyographic (EMG) signals. These techniques aim at recovering unobservable source signals from a set of observations to which they contribute in an unknown way. Many ICA methods have been adapted in order to fit the FECG extraction problem. The multireference adaptive noise cancelling (MRANC) method issued from the adaptive filtering solution by Widrow et al [4] and its adaptation to the FECG extraction problem is presented in [5]. In this work, the authors compare the results provided by the MRANC method with the ones obtained after application of BSS techniques. A review of recent FECG research work can be found in [6].

In Section II we will present the ICA and multidimensional ICA framework and the orthogonal projection methods and we will give an overview of oblique projection operators. Then, in Section III we will present the model we used to simulate the synthetic FECG data sets, the inherent cyclostationarity property of such signals and its measure, as well as the details of the proposed method and the obtained simulation results. A discussion with perspectives for future research are drawn in Section IV. 


\section{Methodology}

\section{A. The ICA model}

Let $M$ be the number of observed signals $x_{i}(t), i \in[1, M]$ to which contribute $N$ unknown sources $s_{i}(t), i \in[1, N]$, with $N \leq M$. The aim of ICA-based methods is to find the unknown sources that are hidden in the observations. The widely used linear instantaneous noiseless BSS model writes in a matrix form:

$$
\mathbf{x}(t)=\mathbf{A s}(t)
$$

where $t$ stands for time, $\mathbf{x}(t)=\left[x_{1}(t), x_{2}(t), \ldots, x_{M}(t)\right]^{\dagger}$ and $\mathbf{s}(t)=\left[s_{1}(t), s_{2}(t), \ldots, s_{N}(t)\right]^{\dagger}$ denote the mixture vector and the source vector, respectively, with ${ }^{\dagger}$ the transpose operator and with $\mathbf{A}$ an unknown $M \times N$ full rank mixing matrix. The mixture model of eq. 1 is based on the assumption of statistical independence of the source components $s_{i}$.

In the context of FECG signal estimation, the measurement vector $\mathbf{x}(t)$ is a linear mixture of the unknown bioelectric current source signals one wants to estimate, i.e. the maternal contribution (MECG) and the foetal contribution (FECG), but also of other artifacts and noise sources, as mentioned in Section I. Let $\mathbf{B}$ be the inverse of the full rank mixing matrix $\mathbf{A}$; then an estimate of the independent components (ICs) of the source vector $\mathbf{s}$ is:

$$
\hat{\mathbf{s}}(t)=\mathbf{B} \mathbf{x}(t) .
$$

In order to find the de-mixing matrix $\mathbf{B}$, some preprocessing steps are commonly used in ICA methods. These are described in the next subsection.

\section{B. Preprocessing for ICA}

In order to simplify ICA algorithms, the most basic preprocessing is to make $\mathbf{x}$ a zero-mean variable by centring it, i.e. by subtracting its mean value $E\{\mathbf{x}\}$. Another useful strategy which is applied as an ICA preprocessing step is a linear transformation of the observations vector $\mathbf{x}$ called whitening. The aim of the whitening step is to obtain a new vector $\tilde{\mathbf{x}}$ which components are uncorrelated and have unit variance, i.e. the covariance matrix of $\tilde{\mathbf{x}}$ is the identity matrix:

$$
E\left\{\tilde{\mathbf{x}} \tilde{\mathbf{x}}^{H}\right\}=\mathbf{I} .
$$

where ${ }^{H}$ denotes the Hermitian transposition.

A popular method for whitening is the Principal Component Analysis (PCA) [7] which consists in an eigenvalue decomposition of the covariance matrix of the observations
$E\left\{\mathbf{x} \mathbf{x}^{H}\right\}=\mathbf{U} \Lambda \mathbf{U}^{H}$ where $\mathbf{U}$ is the orthogonal matrix of eigenvectors of $E\left\{\mathbf{x x}^{H}\right\}$ and $\Lambda$ is the diagonal matrix of its eigenvalues. Thus, the whitened observations vector writes:

$$
\tilde{\mathbf{x}}=\Lambda^{-1 / 2} \mathbf{U}^{H} \mathbf{A} \mathbf{s}=\tilde{\mathbf{A}} \mathbf{s}
$$

with $\tilde{\mathbf{A}}$ a new orthogonal mixing matrix to estimate with less degrees of freedom than the original one. So, whitening reduces the number of parameters to be estimated but it is also a dimension reduction technique, because one can discard the smallest eigenvalues of the covariance matrix of $\mathbf{x}$ that has as effect the reduction of additive noise in the case of the noisy linear instantaneous BSS model. Many ICA algorithms use this whitening step, like the JADE algorithm [8] which is based on a HOS optimisation criterion.

\section{Multidimensional ICA and orthogonal projections}

Several source subspace separation methods for FECG extraction exist in the literature, e.g. [9], where the authors propose a higher-order singular value decomposition (HOSVD) technique to estimate the underlying source subspaces. Another subspace-based technique has been proposed by Cardoso in [10] and it is known as multidimensional independent component analysis (MICA). The author applied this method to a real 3-channel FECG dataset which served as input to the JADE algorithm. After separation, the estimated ICs have been grouped into two independent source subspaces. The first one is a bi-dimensional subspace corresponding to the MECG and the second one is a one-dimensional subspace corresponding to the FECG signal. Next, using the estimated de-mixing matrix $\mathbf{B}$ (eq. 2) provided by JADE, the author computes the orthogonal projection matrices for each one of the two estimated multidimensional components. Finally, by back-projection to the original input space, the mother and foetal signal contributions to the mixtures are computed.

To illustrate the orthogonal projection, let us consider the case where the MICA decomposition yields two linearly independent component subspaces. The $n_{m}$-dimensional mother's subspace in spanned by the $n_{m}$ columns of the demixing matrix corresponding to the $n_{m}$ highest eigenvalues of the diagonal matrix $\Lambda$ after the preprocessing step, while the foetus' $n_{f}$-dimensional subspace in spanned by the $n_{f}$ columns of the de-mixing matrix corresponding to the next $n_{f}$ smaller eigenvalues, with $n_{m}+n_{f}=M$. Let $\mathbf{A}_{f}$ be a $n_{f} \times M$ matrix composed by the $n_{f}$ columns of the mixing matrix $\mathbf{A}$ that span the foetus's subspace. Then, the orthogonal projector $\Pi_{f}$ onto $V_{f}=\operatorname{Span}\left(\mathbf{A}_{f}\right)$ writes:

$$
\Pi_{f}=\mathbf{A}\left(\mathbf{A}^{H} \mathbf{A}\right)^{-1} \mathbf{A}^{H}
$$


and the contribution of the FECG signal to the observations can be expressed as:

$$
\mathbf{x}_{f}=\tilde{\Pi}_{f} \mathbf{x}, \quad \text { with } \quad \tilde{\Pi}_{f}=\Pi_{f} \times\left(\Pi_{f}+\Pi_{m}\right)^{\#},
$$

where $\Pi_{m}$ and superscript ${ }^{\text {\# }}$ denote the orthogonal projector onto $V_{m}=\operatorname{Span}\left(\mathbf{A}_{m}\right)$ (which is defined similarly to that of $\Pi_{m}$ of eq. 5) and the pseudo-inversion operator, respectively. Definitions of orthogonal projectors in the general case are given in [10].

\section{Oblique projections operators}

Oblique projectors [11] decompose a space in two nonorthogonal subspaces. Orthogonal projection is a special case of oblique projection when the two subspaces are orthogonal. Any projector operator $\mathbf{P}$ is idempotent, i.e. $\mathbf{P}^{2}=\mathbf{P}$, but oblique projection matrices are not symmetric. By defining two basis matrices, the first one spanning the interference subspace (called kernel subspace) and the second one spanning the signal of interest (SoI) subspace (called range subspace), oblique projections may be used to cancel the interferences spanned by the kernel subspace.

With respect to the notations introduced in the previous section and in order to fit the FECG extraction problem, let $\mathbf{A}_{m}$ and $\mathbf{A}_{f}$ be the basis matrices defining the kernel and the range subspaces, respectively. Then the oblique projector $P_{\mathbf{A}_{f}}$ whose range is $\left\langle\mathbf{A}_{f}\right\rangle$ writes in matrix form:

$$
P_{\mathbf{A}_{f}}=\mathbf{A}_{f}\left(\mathbf{A}_{f}{ }^{H} P_{\mathbf{A}_{m}}^{\perp} \mathbf{A}_{f}\right)^{-1} \mathbf{A}_{f}{ }^{H} P_{\mathbf{A}_{m}}^{\perp},
$$

with $P_{\mathbf{A}_{m}}^{\perp}=I-\mathbf{A}_{m}\left(\mathbf{A}_{m}{ }^{H} \mathbf{A}_{m}\right)^{-1} \mathbf{A}_{m}{ }^{H}$ and where ${ }^{\perp}$ stands for the orthogonal complement symbol. Finally, the estimated contribution of the FECG signal to the observations using the oblique projection is:

$$
\mathbf{x}_{f_{o}}=P_{\mathbf{A}_{f}} \tilde{\mathbf{x}}
$$

\section{RESULTS AND ILLUSTRATION With SYNTHETIC MECGS}

\section{A. The synthetic FECG signals}

For our experiments we used the open source ECG Toolbox provided by Sameni [12]. The recordings are obtained from electrodes placed in various locations on the expectant mother's body and are contaminated with noise which is a mixture of real baseline wander, electrode movement noise and EMG artifacts. The mother's heartbeat frequency (MHF) is set to $0.9 \mathrm{~Hz}$ while the foetus' heartbeat frequency
(FHF) is equal to $2.2 \mathrm{~Hz}$ and all data are recorded simultaneously at a sampling rate of $500 \mathrm{~Hz}$. The mixture vector $\mathbf{x}$ is 8-dimensional and as similar BSS schemes based on oblique projection operators seem to perform well in very low signalto-noise (SNR) ratios (e.g. [13]), various datasets have been simulated with different SNR values.

\section{B. The cyclostationarity property and measure}

ECG signals have a pseudo-period, i.e. they are not strictly periodic but cyclostationary at the frequency $\alpha_{0}$ of the heartbeats. The cyclostationarity property of the FECGs have been successfully used in previous work [14]. In order to perform MICA decomposition, the authors also proposed a method based on the Cyclic Coherence (CC) [15] which is a normalised cyclostationarity measure based on the correlation degree between two signals at each frequency value; this same measure is used in this work in order to decide whether a JADE-estimated IC belongs to the FECG rather than to the MECG subspace.

\section{Simulations and results}

In order to compare the estimated FECG contributions to the simulated MECG recordings obtained after application of the MICA decomposition based on orthogonal projections and those yielded by the oblique projection of the whitened data provided by the same ICA algorithm, we established the following simulation procedure:

1. Apply the JADE algorithm to estimate ICs from the synthetic MECG data preprocessed for baseline removal with a moving average filtering algorithm.

2. Compute the $\mathrm{CC}$ for each extracted IC and decide to which independent subspace it belongs.

3. Perform JADE-based MICA decomposition as well as oblique projection of the JADE-whitened data during the preprocessing step.

4. Normalise the estimated FECG contributions to the electrodes obtained in the previous step and compute the residual crosstalk (RCT) between the original FECG signals and the estimated ones.

The RCT is a common performance criterion in BSS and writes (in $d B$ ):

$$
R C T(s)=10 \log _{10} \frac{E\left\{(\hat{s}-s)^{2}\right\}}{E\left\{s^{2}\right\}},
$$

where $\hat{s}$ denotes the estimated contribution of the SoI to one of the channels. The more $s$ and $\hat{s}$ are similar the more RCT 

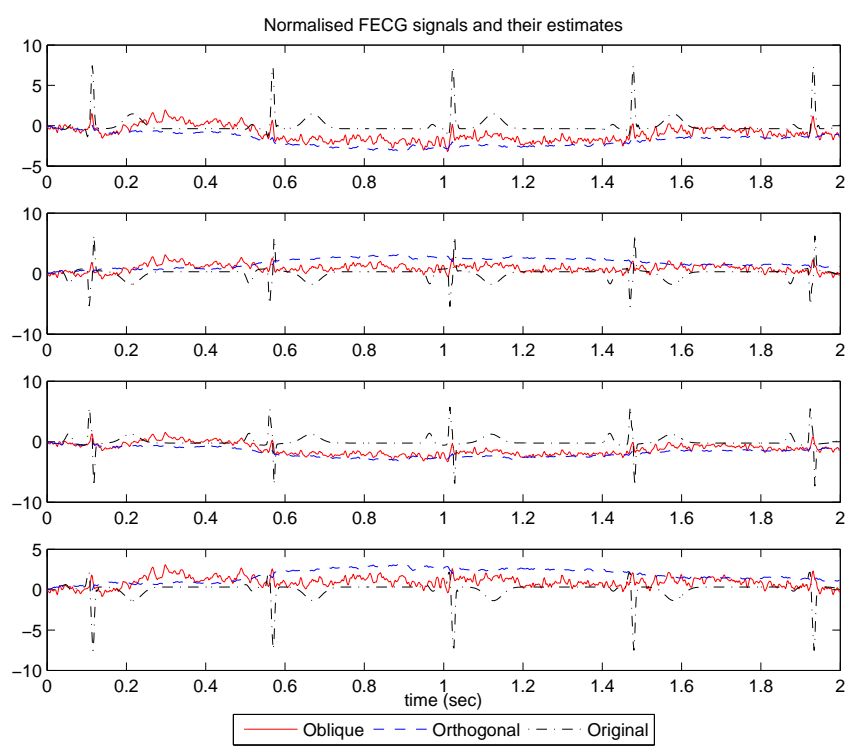

Fig. 1: The original FECGs and their contributions to the 4 first channels (from top to bottom) by oblique projection (solid red plot) and orthogonal projection (dashed blue plot)

tends towards $-\infty$.

For the sake of clarity but also due to lack of space, the estimated FECG contributions to the first 4 channels only are illustrated by Figure 1 in the case of a $0 d b$ SNR value for the synthetic mixture signals. Figure 2 plots the corresponding RCT values for the FECG contribution to all channels; six of the eight extracted FECGs are estimated more accurately in terms of RCT using the oblique projection technique than the orthogonal one. Further experiments were performed with synthetic mixtures contaminated with noise for SNR values in $\{20,10,-10\} d B$. Apart from the $\mathrm{SNR}=20 d B$ case where both methods exhibited similar performance, results obtained with the proposed method were more accurate.

\section{DISCUSSION}

Early results provided by the proposed FECG extraction method based on oblique projection techniques tailored for the BSS/ICA problem are very promising. Current research is focused on the automation of the MICA decomposition step, on the validation of the proposed method on real FECG data sets and on taking into account specific properties of such bioelectric signals.

\section{REFERENCES}

1. Longini R L, Reichert T A, Yu J M C, Crowley J S. Near-Orthogonal Basis Functions: A Real Time Fetal ECG Technique IEEE Transactions

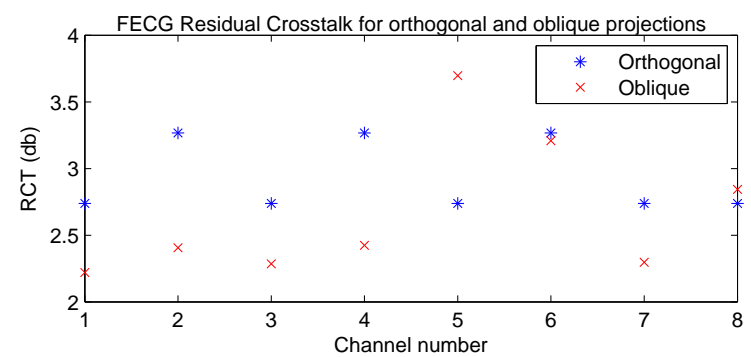

Fig. 2: Residual Crosstalk values in $d B$ (y-axis) for the extracted FECG contributions to the 8 channels (x-axis) after oblique (red cross marks) and orthogonal (blue star marks) projections

on Biomedical Engineering. 1977;BME-24:39-43.

2. Herault J, Jutten C, Ans B. Détection de grandeurs primitives dans un message composite par une architecture neuromimétique en apprentissage non supervisé in Actes 10ème Colloque GRETSI(Nice, France):1017-1022 1985 .

3. Comon P. Independent Component Analysis, a New Concept Signal Processing, Special Issue on Higher Order Statistics. 1994;36(3):287314.

4. Widrow B, Glover J R, McCool J M, et al. Adaprive Noise Cancelling: Principles and Applications Proceedings of the IEEE. 1975;63:16921716.

5. Zarzoso V, Nandi A K. Noninvasive Foetal Electrocardiogram Extraction: Blind Separation Versus Adaptive Noise Cancellation IEEE Transactions on Biomedical Engineering. 2001;48(1):12-18.

6. Sameni R, Clifford G D. A Review of Fetal ECG Signal Processing Issues and Promising DIrections The Open Pacing, Electrophysiology \& Therapy journal. 2010;3:4-20.

7. Comon P, Jutten C. Handbook of Blind Source Separation: Independent Component Analysis and Applications. Academic Press 2010.

8. Cardoso J F, Souloumiac A. Blind beamforming for non Gaussian signals in Radar and Signal Processing, IEE Proceedings F;140(6):3623701993.

9. Lathauwer L De, Moor B De, Vanderwalle J. Fetal Electrocardiogram Extraction by Source Subspace Separation in Proceedings IEEE Signal Processing / Athos Workshop on Higher-Order Statistics:134-138 1995.

10. Cardoso J F. Multidimensional independent component analysis in Proceedings of the 1998 IEEE International Conference on Acoustics, Speech and Signal Processing;4:1941-1944 1998.

11. Behrens R T, Scharf L L. Signal Processing Applications of Oblique Projection Operators IEEE Transactions on Signal Processing. 1994;42:1413-1424.

12. Sameni R. Open Source ECG Toolbox (OSET) 2006.

13. Zhang Y Q, Zhang B Cao Q Y, Chen X. Blind Signal Separation Using Oblique Projection Operators Method in Proceedings IWCMC'10:5605642010.

14. Haritopoulos M, Capdessus C, Nandi A K. Foetal PQRST Extraction from ECG Recordings using Cyclostationary-Based Source Separation Method in 32 Annual International Conference of the IEEE EMBS:1910-1913 2010.

15. Haritopoulos M., Roussel J., Capdessus C., Nandi A.K.. Cyclostationarity-Based Estimation of the Foetus Subspace Dimension from ECG Recordings in XIII Mediterranean Conference on Medical and Biological Engineering and Computing 2013 (Romero Laura M. Roa. , ed.);41 of IFMBE Proceedings:726-729Springer 2014. 